\title{
PERCEPCIÓN DE CUIDADO HUMANIZADO DE ENFERMERÍA DESDE LA PERSPECTIVA DE USUARIOS HOSPITALIZADOS ${ }^{1}$
}

\author{
PERCEPTION OF HUMANIZED NURSING CARE \\ BY HOSPITALIZED USERS
}

\author{
Pablo Monje V. \\ Paulina Miranda C. ${ }^{* *}$ \\ JésSica Oyarzún G. ${ }^{* * *}$ \\ Fredy Seguel P. ${ }^{* * *}$ \\ ELIZABETh Flores G. ${ }^{* * * *}$
}

\begin{abstract}
RESUMEN
Objetivo: Determinar la percepción que tienen los pacientes hospitalizados en Servicio de Medicina Adulto de un hospital de alta complejidad, del cuidado humanizado que otorgan los profesionales de Enfermería y factores relacionados. Material y métodos: Estudio de corte transversal, correlacional, en una muestra de 171 pacientes hospitalizados en el servicio de medicina, durante el año 2014. Se aplicó una encuesta sociodemográfica y la escala Percepción de Comportamientos de Cuidado Humanizado de Enfermería (PCHE), segunda versión, adecuada transculturalmente a Chile. Resultados: 54,4\% de los pacientes hospitalizados fueron de sexo femenino, media de edad de 56,2 años y 6,95 días de hospitalización, diagnóstico de ingreso de la mayoría fue enfermedad del sistema circulatorio (33,9\%). El 86\% de los pacientes hospitalizados considera que siempre recibe trato humanizado, $11,7 \%$ casi siempre, $1,8 \%$ algunas veces y un $0,6 \%$ nunca percibió un trato humanizado. Las cualidades en la entrega de cuidado humanizado por el profesional de enfermería que destacan los pacientes son las relacionadas a la categoría "Cualidades del hacer", es decir, perciben que son capaces de identificar sus necesidades, mantienen trato cordial, tienen buena disponibilidad cuando se les requiere y los educan. No existe asociación estadísticamente significativa entre el nivel de percepción de trato humanizado y las variables sociodemográficas. Conclusión: Los/as enfermeros/as de un hospital de alta complejidad respetan la dignidad humana al entregar cuidados humanizados, principalmente por la forma en que proporcionan los cuidados día a día. El estudio permite retroalimentación positiva al profesional que labora en la unidad, fortalecer su desempeño y la motivación para cuidar.
\end{abstract}

Palabras clave: Enfermería, cuidado de enfermería, humanización de la atención, relaciones enfermero-paciente.

\begin{abstract}
Objective: To determine the perception of hospitalized patients in a high complexity hospital regarding the provision of humanized care by nursing professionals and related factors. Methods: Cross-sectional correlational study, a demographical survey and Perception Behavior Scale of Humanized Nursing Care (PCHE), second

\footnotetext{
${ }^{1}$ Artículo derivado de la Tesis "Percepción de cuidado humanizado en el servicio de Medicina Adulto, en un hospital de alta complejidad, tercer trimestre 2014" presentada como parte de los Requisitos para optar al grado de Licenciado en Enfermería.

* Licenciado en Enfermería. Universidad Austral de Chile. Email: pablomonjevera@gmail.com

** Licenciada en Enfermería. Universidad Austral de Chile. Email: paulina.miranda.c@gmail.com

${ }^{* * *}$ Licenciada en Enfermería. Universidad Austral de Chile. Email: jessoyarzun@gmail.com

${ }^{* * * *}$ Doctor en Enfermería. Académico Universidad Austral de Chile. Email: fredyseguel@uach.cl

***** Magíster en Enfermería. Académica Universidad Austral de Chile. Email:idaflores@uach.cl. Autor de correspondencia.
} 
version cross-culturally adequate for Chile, was applied to a sample of 171 hospitalized patients during 2014. Results: $54.4 \%$ of hospitalized patients were female, mean age 56.2 years, and with a mean 6.95 days of hospitalization, admission diagnosis was mostly circulatory system disease (33.9\%). 86\% of hospitalized patients considered they always receive humane treatment, $11.7 \%$ almost always, $1.8 \%$ sometimes and $0.6 \%$ perceived never receiving humane treatment. The qualities in the delivery of humanized care by nursing professionals highlighted by patients are related to the category "Qualities of doing", in other words, patients perceive that the nurses are able to identify their needs, maintain cordiality, show availability when they are required, and are polite educate patients. No statistically significant association between perceived level of humane treatment and sociodemographic variables was found. Conclusion: The nurses in a high complexity hospital respect human dignity by delivering humanized care, mainly due to the way they provide day to day care. This study provides positive feedback to the professional working in the unit, strengthens their performance and motivation for caring.

Key words: Nursing, nursing care, humanization of assistance, nurse-patient relations.

Fecha recepción: 12/06/15 Fecha aceptación: 21/12/17

\section{INTRODUCCIÓN}

Enfermería, entendida como ciencia y arte del cuidado, se destaca por la entrega de atención desde una perspectiva biopsicosocial, siendo fundamental las relaciones que se establecen con los pacientes (1). La disciplina de Enfermería considera el cuidado como eje de su quehacer, en la atención y prestación de servicios sanitarios en salud, con un compromiso en la entrega de cuidados no observado en otra disciplina (2). Cuidar es la base moral sobre la cual se desarrollan las obligaciones profesionales y éticas; constituye el fundamento a partir del cual se rige la praxis profesional en Enfermería (3).

Según Kérouack (4), los cuidados de enfermería se caracterizan por ser el resultado del análisis y pensamiento crítico, polivalentes, con un alto nivel de competencias técnicas, creativos, flexibles, pero también, de una gran sensibilidad en el trato humano de los individuos que se encuentran en situaciones de salud-enfermedad.

Sin embargo, el escenario en salud es cambiante, hay un importante desarrollo tecnológico de las comunicaciones, cambios demográficos y epidemiológicos, que provoca cambios en la entrega de los cuidados de enfermería, por lo que es necesario reflexionar sobre la forma en que establecemos la relación con el usuario, desde la humanización de la praxis. (5). Es paradójico que, en este escenario de gran desarrollo científico en el ámbito de la medicina, con progresos en tecnología de punta, los pacientes aún se sientan desprotegidos ante el sistema de salud y muestren insatisfacción en el trato que se les otorga (6). Los integrantes del equipo de salud deben saber equilibrar en sus competencias profesionales las destrezas y pericias técnicas y el sentido humano en la atención, para no terminar "cosificando" al paciente; tener una visión integral de éste, entender que es una persona frágil y angustiada por la situación de salud que está viviendo, requiere valorar sus sentimientos y riqueza espiritual y adoptar una adecuada actitud de ayuda, además de gestionar para lograr una atención humanizada (7).

Watson, autora de la "Teoría del Cuidado Humano", sostiene que, ante la posible deshumanización de la atención en salud por cambios de estructura, nuevos modelos y tecnologías, es necesario el rescate del aspecto humano, espiritual y transpersonal, por parte de los profesionales de Enfermería (5). Por otra parte, la gestión del cuidado que se entiende como "el ejercicio de competencias 
personales, profesionales e instrumentales que permiten organizar, coordinar y articular los cuidados en los diferentes niveles de atención, asegurando con ello la continuidad y eficacia de los mismos", tiene como finalidad entregar cuidados de calidad a las personas, tomando en cuenta sus derechos y situación actual de salud (8).

La reforma de la salud chilena, en el año 2012 establece la Ley 20.584 que regula los Derechos y Deberes que tienen las personas en relación con acciones vinculadas a su Atención de Salud (9), en la que se establecen dos pilares fundamentales en la entrega de servicios sanitarios: enfoque de integralidad, fortalecer la gestión clínica y del cuidado; en concordancia con lo anterior, el Código de Ética del Colegio de Enfermeras de Chile establece directrices en relación al trato digno: la enfermera, al entregar cuidados debe respetar los derechos humanos, valores, costumbres y creencias espirituales, verificando en todo momento que las personas reciban información suficiente para aceptar o rechazar los cuidados y tratamientos; resguardando el secreto profesional de cualquier información confidencial, siempre y cuando su silencio no afecte la vida o salud del individuo o de otras personas (10). En Chile, desde el año 2013 es exigible la garantía de Calidad y Seguridad de los Pacientes².

Los cuidados de enfermería, por tanto, deben estar en concordancia con el quehacer cotidiano, donde se trata con personas que poseen sentimientos, dudas y temores respecto al acto sanitario y al continuo salud-enfermedad; por este motivo, es necesaria la incorporación de instrumentos que midan la calidad de la atención desde la perspectiva humanizada (11).

Diversos autores han conceptualizado el cuidado humanizado, centrándose en el enfoque integral y holístico que debe tener la entrega de cuidados, destacando que debe

${ }^{2}$ Ley 19.966 Régimen de Garantías Explícitas en Salud y sus Decretos 2005-2007. Ministerio de Salud, Chile. haber un equilibrio entre los conocimientos de la ciencia y los valores, por lo que los principios de la bioética deben regir el actuar de todo profesional de enfermería y la base moral y filosófica del cuidar $(12,13)$. Watson, a partir de sus experiencias personales y profesionales, elabora conceptos teóricos e identifica la humanización como un componente elemental en la entrega de cuidados (5). Afirma que la conceptualización de los seres humanos es el potencial metafísico para la autocuración y trascendencia a niveles superiores de conciencia $(14,15)$, sentando las bases de su "Teoría del Cuidado Humano", fundada en la filosofía y la ética (16). Esta filosofía y teoría entrega una forma de combinar el ambiente sanitario de hoy, altamente técnico y consciente de los costos, con las prácticas más humanas del cuidado (16). Además, incorpora conceptos de humanización de cuidados, altruismo, toma de valores, cultivo de la sensibilidad y establecimiento de relación de ayuda entre los individuos, que avalan el carácter sociopsicobiológico de la disciplina, mencionando que el cuidado humano está basado en valores como la bondad, preocupación y amor a sí mismo y a los demás (14). La relación del cuidado transpersonal se basa en la capacidad del enfermo de tener una visión objetiva de su situación y la capacidad de los profesionales de enfermería, de mostrar una genuina preocupación por la persona en todos los ámbitos del ser, creando una conexión espiritual, que facilite el afrontamiento de la enfermedad; asumiendo una posición de trascendencia frente al sufrimiento que permita lograr la armonía interior (17).

Por otro lado, la dignidad de las personas es el principio rector de la bioética; la persona tiene derecho a recibir un trato digno, de calidad y con un énfasis integral acorde a las demandas sanitarias de la población; cada integrante del equipo debe proveer las condiciones idóneas para la salud; enfermería debe retomar la mirada en la cual el "otro" es el centro del cuidado, gestionar los recursos, 
mejorar la infraestructura, respetar la intimidad y tomar conciencia de la vulnerabilidad de los usuarios, es decir, crear instancias de humanización y controlar aquellos aspectos que influyen negativamente $(6,18-20)$. En la década del 70' a nivel mundial se manifiesta la satisfacción usuaria como una medida del resultado de la interacción entre los profesionales de la salud y el paciente (18). Según Santacruz-Bravo, en la actualidad la atención en salud tiene como centro de su interés al paciente, lo que exige tener conciencia humanista para dar respuesta a los derechos de éste, utilizando la ética para su logro (21).

Existen diversos estudios que describen la experiencia de recibir un cuidado humanizado de enfermería, Alvis et al. (22), basado en la Teoría del Cuidado Humano de Jean Watson y a partir de la interacción del cuidador-ser cuidado, definen 9 comportamientos del cuidado humanizado: sentimientos del paciente, características de la enfermera, dar apoyo emocional, dar apoyo físico, cualidades del hacer de la enfermera, proactividad, empatía, priorizar al ser cuidado y disponibilidad para la atención.

A partir de la investigación de Alvis et al., Rivera y Triana (23) realizaron en Bogotá el estudio "Percepción de comportamientos de cuidado humanizado de Enfermería en pacientes hospitalizados en la Clínica del Country", cuyo resultado fue la elaboración del instrumento "Percepción de comportamientos de cuidado humanizado de Enfermería" (PCHE). Posteriormente, González y Quintero ${ }^{3}$ utilizando el instrumento PCHE, primera versión, encontraron que la mayoría de los pacientes siempre percibieron comportamiento de cuidado humanizado (73\%).

Por otra parte, un estudio en Perú concluyó que existe una baja percepción del cuidado

\footnotetext{
${ }^{3}$ González J, Quinteros D. Percepción del cuidado Humanizado en pacientes que ingresan al servicio de hospitalización de urgencias de ginecobstetricia de una institución de II y III nivel de atención. [Trabajo de grado para optar al título de Enfermera]: [Bogotá]: Facultad de Enfermería, Pontificia Universidad Javeriana; 2009. 101 p.
}

humanizado, evidenciando una atención de baja calidad (24), a diferencia de un estudio realizado en Colombia en el que se evidenció que el $70 \%$ de los pacientes siempre perciben comportamientos de cuidado humanizado, siendo la categoría "priorizar al ser cuidado" la mejor evaluada y "proactividad" la peor evaluada ${ }^{4}$.

Por su parte, un estudio chileno que utilizó PCHE segunda versión señala que la mayoría de las personas hospitalizadas en un hospital público de Valparaíso perciben que siempre recibió trato humanizado, las categorías con mayor percepción fueron: cualidades del hacer $(95,75 \%)$, priorizar al ser cuidado $(94,93 \%)$, apoyo físico $(94,20 \%)$. Las Categorías de menor percepción son apoyo emocional $(92,75 \%)$, proactividad $(90,58 \%)$ y empatía $(90,58 \%)^{5}$. Sin embargo, otros autores también encontraron que la percepción de calidad del cuidado de enfermería se asocia más a las habilidades técnicas que a los comportamientos de trato humanizado (23).

Considerando el cuidado como deber moral, centro de la atención de la disciplina de enfermería, un mandato en la legislación chilena, que la calidad en la atención debe estar garantizada y ser un pilar de la reforma de salud, surge la necesidad de conocer desde la perspectiva de los pacientes: ¿Cuál es la percepción que poseen respecto al cuidado humanizado entregado por los profesionales de Enfermería?

El estudio tuvo como objetivo determinar la percepción que tienen los pacientes hospitalizados en el Servicio de Medicina Adulto de un hospital de alta complejidad, del cui-

${ }^{4}$ Acosta A, Mendoza C, Morales K, Quiñones A. Percepción del paciente hospitalizado sobre el cuidado humanizado brindado por Enfermería en una IPS de tercer nivel. Cartagena 2013. [Trabajo de grado para optar al título de Enfermero profesional]: [Cartagena de Indias]:Facultad de Ciencias de la salud Corporación Universitaria Rafael Núñez; 2013. 154 p.

${ }^{5}$ Acosta C, Cortez Y, Acevedo K, Cantarutti C. Percepción sobre el cuidado humanizado proporcionado por la enfermera en atención cerrada. Poster presentado en: VIII Congreso Internacional de Estudiantes de Enfermería de Chile; 29 jul al 4 agosto 2013; Concepción, Chile. 
dado humanizado que otorgan los profesionales de enfermería y factores relacionados.

Los resultados permitirán la retroalimentación positiva del profesional que labora en la unidad, fortalecer su desempeño y la motivación para cuidar. Además, contribuirán a potenciar la humanización del cuidado y el respeto a la dignidad de los usuarios.

\section{MATERIAL Y MÉTODO}

Investigación de corte transversal y correlacional. La población de estudio fue una muestra representativa, aleatoria simple de 171 pacientes, que corresponde a una población aproximada de 603 pacientes hospitalizados, con una confianza del $95 \%$ y un error estadístico del 5\%, en el Servicio de Medicina Adulto de un hospital de alta complejidad del sur de Chile durante los meses de julio, agosto y septiembre de 2014. Se incluyeron mayores de 15 años, tiempo de permanencia de 1 o más días, en el caso de usuarios menores de 18 años que aceptaron participar, se solicitó Consentimiento de sus tutores legales (5 personas). Se excluyeron a aquellas personas con compromiso de conciencia que les impidió responder las encuestas, con alteración o limitación cognitiva.

Para la recolección de datos se utilizaron 2 instrumentos, que fueron aplicados durante la hospitalización: 1. Percepción de Comportamientos de Cuidado Humanizado de Enfermería (PCHE); 2. Encuesta Sociodemográfica de elaboración propia, que incluye: sexo, edad, número de días de hospitalización, nivel de escolaridad, grupo diagnóstico, religión y etnia.

El Instrumento PCHE, segunda versión, posee una validez de contenido 0,83 y una validez facial de 0,78 (25). Para su utilización en el presente estudio se utilizó la adecuación transcultural al contexto chileno ${ }^{6}$. Este Ins-

${ }^{6}$ Acevedo K, Acosta C, Cantarutti C, Cortez G, Menares trumento consta de 32 ítems distribuidos en 6 categorías, se utiliza una escala de Likert de 4 puntos, en donde 1 corresponde a nunca, 2 algunas veces, 3 casi siempre y 4 siempre, con puntajes por encuesta de 32 a 128 (25). Las 6 categorías incluidas son:

- Dar Apoyo Emocional: compuesta por los ítems $\mathrm{N}^{\text {os }} 6,7,8,21,25$ y 26 , que apuntan a cómo el paciente percibe la relación con el profesional de Enfermería a través de un lazo de confianza.

- Dar Apoyo Físico: compuesta por los ítems 3, 4, 18 y 27, considera acciones de la enfermera relacionadas al contacto físico respetuoso, respuesta a la necesidad de acompañamiento, alivio del dolor y comodidad del paciente.

- Empatía: capacidad del profesional de Enfermería de establecer una relación empática con el enfermo, de ponerse en su lugar, favoreciendo la expresión de sentimientos, distribuida en los ítems 9, 12, 15 y 24.

- Cualidades del hacer de la/el Enfermera/o: cualidades en la entrega de cuidado del profesional de enfermería, es decir, su disponibilidad, trato cordial, que es capaz de identificar sus necesidades y educarlo, especificados en los ítems $2,5,10,14,22$, 23, 28 y 32.

- Proactividad: compuesta por los ítems $11,13,19,20$ y 30 , comprende acciones de la enfermera que indican su grado de iniciativa, capacidad de dar respuesta a las interrogantes del paciente y su familia y hacerlos partícipes de su recuperación.

- Priorizar al ser cuidado: acciones de enfermería relacionadas al sentimiento de "ser valorado" por el profesional como ser único con necesidades diferentes, distribuidas en los ítems 1, 16, 17, 29 y 31.

C, Uribe P, Bettancourt L. Percepción sobre el cuidado humanizado proporcionado por profesionales de Enfermería en atención cerrada, Valparaíso, 2013. [Tesis para optar al grado de Licenciado en Enfermería]: [Valparaíso]: Universidad de Valparaíso; 2013. 
Los rangos para cada categoría son: 104,1 - 128 puntos: siempre; 80,1 - 104 puntos: casi siempre; 56,1 - 80 puntos: algunas veces; 32 - 56 puntos: nunca se percibe una relación de cuidado humanizado en las(os) enferme$\operatorname{ras}(\mathrm{os})$.

Para el procesamiento de datos se realizaron análisis estadísticos descriptivos de las variables de estudio mediante medidas de tendencia central (media, medianas, moda) y de variabilidad (desviación estándar), a fin de determinar la percepción del cuidado humanizado otorgado por profesionales de enfermería a los usuarios hospitalizados en el Subdepartamento de Medicina Adulto del hospital de alta complejidad, además se realizaron pruebas de correlación, Prueba de Spearman, U-Mann Whitney y Chi cuadrado.

Los datos fueron analizados con el programa SPSS de Windows, versión 11.5. La variable dependiente fue Nivel de Percepción de Cuidado Humanizado: Percepción Cuidado Humanizado Global (siempre, casi siempre, algunas veces, nunca), Percepción Cuidado Humanizado por categorías dar apoyo emocional, dar apoyo físico, empatía, cualidades del hacer de la enfermera, proactividad, priorizar al ser cuidado (siempre, casi siempre, casi nunca, nunca). Y las variables independientes fueron: sexo, religión, etnia, nivel de escolaridad, grupo diagnóstico, edad, No de días de hospitalización, años de estudio.

El estudio contó con la autorización de los autores para la utilización del instrumento Percepción de Comportamientos de Cuidado Humanizado de Enfermería (PCHE), de la dirección de Gestión de Cuidado del Hospital y del Comité de Ética del Servicio de Salud correspondiente.

\section{RESULTADOS}

El perfil de los usuarios del servicio de medicina adulto encuestados se caracteriza por una media de edad de 56,2 años, con una mediana de 60 años, un promedio de 6,95 días de hospitalización, con una mediana de 4 días. El mínimo de días de hospitalización fue de 1 y el máximo de días de hospitalización fue de 50 días (Tabla 1).

El 54,4\% correspondía a mujeres, el diagnóstico de mayor frecuencia fue de enfermedades circulatorias $(33,9 \%)$, seguido por las enfermedades de la sangre y órganos hematopoyéticos $(18,1 \%)$ y respiratorias $(10,5 \%)$. Más de la mitad de los pacientes sólo alcanzaron escolaridad básica incompleta y el 8,7\% tenía escolaridad superior (técnico superior y universitaria completa). La mayoría profesa la religión católica (52\%) y evangélica (27,5\%), sólo el $11,7 \%$ se consideran parte de la etnia mapuche u otras etnias.

Respecto a la percepción de cuidado humanizado, los resultados muestran que el promedio del puntaje global obtenido fue de 116,65 pts., es decir siempre se percibe cuidado humanizado. El puntaje mínimo obtenido fue 50 pts., correspondiente a la categoría nunca se percibe cuidado humanizado. De acuerdo con la percepción de cuidado humanizado por categorías, la distribución de las medias mostró que todas las categorías son percibidas como siempre (Tabla 2).

El puntaje global de la Percepción de Cuidado Humanizado en Enfermería muestra que la mayoría de los pacientes encuestados $(86 \%)$ siempre perciben trato humanizado por parte de los profesionales de enfermería y que sólo el 0,6\% de los entrevistados nunca percibió un trato humanizado durante su estadía hospitalaria (Tabla 3). 
Tabla 1. Descripción de las variables edad y número de días de hospitalización.

\begin{tabular}{lcccccc}
\hline & Media & Mediana & Moda & Desv. típ. & Mínimo & Máximo \\
\hline Edad & 56,2 & 60 & 67 & 18,85 & 15 & 88 \\
No días hospitalización & 6,95 & 4 & 1 & 8,45 & 1 & 50 \\
\hline
\end{tabular}

Tabla 2. Distribución de puntajes según categorías de PCHE y puntaje global.

\begin{tabular}{lcccccc}
\hline $\begin{array}{l}\text { Percepción de cuidado } \\
\text { humanizado de enfermería }\end{array}$ & Media & Mediana & Moda & Desv. típ. & Mínimo & Máximo \\
\hline Puntaje Global & 116,65 & 121 & 128 & 13,303 & 50 & 128 \\
Dar apoyo emocional & 21,7 & 23 & 24 & 3,16 & 6 & 24 \\
Dar apoyo físico & 14,56 & 16 & 16 & 2,22 & 4 & 16 \\
Empatía & 14,23 & 15 & 16 & 1,99 & 7 & 16 \\
Cualidades del hacer & 28,97 & 30 & 32 & 3,59 & 12 & 32 \\
Proactividad & 18,35 & 20 & 20 & 2,85 & 5 & 20 \\
Priorizar al ser cuidado & 18,41 & 20 & 20 & 2,13 & 11 & 20 \\
\hline
\end{tabular}

Tabla 3. Distribución de la percepción de cuidado humanizado de enfermería (PCHE) según categorías y puntaje total.

\begin{tabular}{lccccccccccc}
\hline & \multicolumn{8}{c}{ Distribución de la percepción de cuidado humanizado de enfermería } \\
\cline { 2 - 11 } Categorías & \multicolumn{3}{c}{ Nunca } & \multicolumn{3}{c}{ Algunas veces } & Casi siempre & Siempre & \multicolumn{3}{c}{ Total } \\
\cline { 2 - 11 } & $\mathbf{f}$ & $\%$ & f & $\%$ & f & $\%$ & f & $\%$ & f & $\%$ \\
\hline Dar apoyo emocional & 3 & 1,8 & 4 & 2,3 & 22 & 12,9 & 142 & 83,0 & 171 & 100 \\
Dar apoyo físico & 3 & 1,8 & 6 & 3,5 & 37 & 21,6 & 125 & 73,1 & 171 & 100 \\
Empatía & 2 & 1,2 & 4 & 2,3 & 58 & 33,9 & 107 & 62,6 & 171 & 100 \\
Cualidades del hacer & 2 & 1,2 & 3 & 1,8 & 30 & 17,5 & 136 & 79,5 & 171 & 100 \\
Proactividad & 4 & 2,3 & 4 & 2,3 & 20 & 11,7 & 143 & 83,6 & 171 & 100 \\
Priorizar el cuidado & 0 & 0,0 & 3 & 1,8 & 22 & 12,9 & 146 & 85,4 & 171 & 100 \\
Puntaje Global & 1 & 0,6 & 3 & 1,8 & 20 & 11,7 & 147 & 86,0 & 171 & 100 \\
\hline
\end{tabular}

Categoría Dar apoyo emocional: el 83\% responde que siempre se ha sentido apoyado emocionalmente por el profesional de enfermería durante su estadía hospitalaria, mientras que 22 personas $(12,9 \%)$ contestan casi siempre, de igual forma, sólo 4 personas
$(2,3 \%)$ casi nunca y el 1,8\% responde nunca. Categoría Dar apoyo físico: el 73,1\% (125 personas) respondió siempre, mientras que el 21, 6\% mencionó casi siempre, el 3,5\% de los participantes afirma que casi nunca siente apoyo físico, en tanto el 1,8\% nunca. 
Categoría Empatía: se observa que el 62,6\% manifestó que siempre perciben un trato humanizado empático, mientras que un 33,9\% casi siempre, el 2,3\% casi nunca y el 1,2\% nunca.

Categoría Cualidades del hacer de la/el Enfermera/o: un 79,5\% del total siempre percibe las cualidades en la entrega de cuidado del profesional de enfermería, es decir, su disponibilidad, trato cordial, que es capaz de identificar sus necesidades y educarlo, un 17,5\% casi siempre, $1,8 \%$ casi nunca y el $1,2 \%$ nunca.

Categoría Proactividad: el 83,6\% siempre perciben proactividad en el profesional de Enfermería, el 11,7\% casi siempre, el 2,3\% casi nunca, 2,3\% nunca.

Categoría Priorizar al ser cuidado: 146 personas de las encuestadas $(85,4 \%)$ refiere que el profesional de Enfermería siempre prioriza el ser cuidado durante su atención, un 12,9\% refiere que casi siempre se da esta situación y un $1,8 \%$ refiere que casi nunca se prioriza al ser cuidado.

Para evaluar la significancia de la asociación entre la variable dependiente y las independientes se utilizó el estadígrafo Chi cuadrado, es así como entre puntaje global y nivel de escolaridad existe asociación estadísticamente significativa $\left(c^{2}=78,890, p=0,000\right)$, sin embargo, la mayoría de los pacientes contestaron siempre y en las categorías de nunca, algunas veces y casi siempre prácticamente no hubo respuesta, lo que puede influenciar el resultado de la prueba de Chi cuadrado.

Se constató que la asociación entre las variables percepción de cuidado humanizado y sociodemográficas, edad $(\mathrm{Rho}=-0,033, \mathrm{p}=$ $0,670)$, sexo $(U=3252, p=0,710)$ y No días de hospitalización $(\mathrm{Rho}=-0,111, \mathrm{p}=0,151)$, no fue significativa.

\section{DISCUSIÓN Y CONCLUSIÓN}

La forma de llevar a cabo el cuidado es a través del desarrollo de un vínculo enfermeropaciente. Este vínculo está dado por diversas características del profesional, las habilidades técnicas, el conocimiento y las actitudes como la empatía, respeto y la autenticidad (26). Según el instrumento PCHE, este vínculo es evaluado de manera satisfactoria por los pacientes del Servicio de Medicina, quienes perciben que siempre se otorga un cuidado humanizado en la atención de Enfermería $(5,26)$. La bibliografía revisada confirma lo encontrado en este estudio $(3,23)$, mostrando que los pacientes perciben que los profesionales invierten tiempo para establecer algún vínculo más humano, además de la aplicación de técnicas o procedimientos. Resalta un estudio chileno realizado en Valparaíso ${ }^{7}$, que refiere diferencias no significativas en relación con la edad y sexo, que es concordante con esta investigación.

Contrario a los hallazgos de Romero et al. (27) que mencionan que no existe relación entre la variable independiente nivel de escolaridad de los usuarios y percepción de cuidado humanizado, en este estudio se demostró una asociación estadísticamente significativa entre las variables.

Por otro lado, la categoría "Cualidades del hacer" presentó mayor percepción de cuidado humanizado concordante con otros autores (25) y con el trabajo realizado en Valparaíso. En resumen, los diversos estudios realizados reflejan concordancia en sus resultados con esta investigación, con una respuesta positiva o satisfactoria de la percepción de cuidado humanizado en enfermería.

Es necesario resaltar que el instrumento PCHE evalúa de manera indirecta el cumplimiento de tres derechos incluidos en la Ley 20.584 que regula los derechos y deberes que tienen las personas en relación con acciones

\footnotetext{
${ }^{7}$ Acevedo et al. cit. n. 6.
} 
vinculadas a su atención de salud: derecho a un trato digno (ítems 4, 5, 12 y 24); derecho a la compañía, asistencia espiritual y a la atención con pertinencia cultural (ítems 17 y 23); derecho a la autonomía (ítems 19 y 29). Todos los ítems relacionados fueron evaluados en la categoría "siempre" en la escala global.

El estudio ratifica que Enfermería sustentada en sus bases disciplinares tiene como esencia la entrega de cuidados humanizados, oportunos y de calidad, que se convierten en las principales características de los profesionales de Enfermería. Esto, sumado al actual énfasis a nivel ministerial y en muchas Escuelas de Enfermería a nivel nacional e internacional, en las que se ha enfatizado el cuidado integral de las personas y no sólo suplir sus necesidades físicas, hace que los pacientes perciban un mejor trato por parte de los profesionales.

La importancia de la entrega de cuidados cálidos y humanos por el profesional de Enfermería radica en que permite a las personas que han sufrido una interrupción en su cotidiano vivir y han sido sacados de su entorno sentirse acompañados para enfrentar el proceso de salud-enfermedad.

El estudio realizado permite una retroalimentación positiva para el personal que labora en servicios de hospitalización, fortaleciendo la identidad y el posicionamiento de la profesión y diferenciación con las demás disciplinas. Deja abierta la posibilidad de un abordaje desde el paradigma cualitativo, que permitiría abarcar otros ámbitos.

Agradecimientos: a Sonia Espitia, Álvaro Triana y Luz Nelly Rivera. Departamento de Enfermería, Clínica del Country. Facultad de Enfermería, Universidad Nacional de Colombia. Bogotá, Colombia, 2010. Quienes facilitaron el instrumento PCHE $2^{\text {a }}$ versión.

\section{REFERENCIAS}

1. Zárate R. La Gestión del Cuidado de Enfermería. Index Enferm. 2004; 13(44-45): 42-46.

2. Ariza C, Daza de Caballero R. Calidad del Cuidado de Enfermería al paciente hospitalizado en un hospital de tercer nivel en Bogotá. Actual Enferm. 2005; 8(3): 8-14.

3. Pellegrino E. The caring ethics. En Bishop $\mathrm{AH}$, Scudder JR, ed. Caring, Curing, Coping: Nurse, Physician, Patient Relationships. Tuscaloosa, Alabama: The University Alabama Press; 1985.

4. Kérouack S, Pepin J, Ducharme F, Duquette A, Major F. El Pensamiento Enfermero. España: Elsevier Doyma; 1996. p. 2-3.

5. Urra E, Jana A, García M. Algunos aspectos esenciales del pensamiento de Jean Watson y su teoría de cuidados transpersonales. Cienc. enferm. 2011; XVII(3): 11-22.

6. Correa M. La humanización de la atención en los servicios de salud: un asunto de cuidado. Rev Cuid. 2016; 7(1): 122731.

7. Quiala M. Enfermería, formación en valores. Bioética [Internet]. 2013 [citado 01 mar 2014]; 13(1): 20-25. Disponible en: http://www.cbioetica.org/revista/131/131-2025.pdf

8. Milos P, Bórquez B, Larraín A. La "Gestión del Cuidado" en la legislación chilena: Interpretación y alcance. Cienc. enferm. 2010; XVI(1): 17-29.

9. Ley No 20.584. Regula los derechos y deberes que tienen las personas en relación a acciones vinculadas a su atención en salud. Ministerio de Salud, Subsecretaría de Salud Pública (Abr. 04, 2012).

10. Colegio de Enfermeras de Chile. Código de Ética del Colegio de Enfermeras de Chile [Internet]. Santiago (CL): Colegio de Enfermeras de Chile; 2008 [ci- 
tado 01 mar 2014]. 16 p. Disponible en: http://enfermeriajw.com/wp-content/ uploads/2012/08/Coodigo-de-Etica-Colegio-de-Enfermeras-de-Chile.pdf.

11. Moreno M. Humanización del Cuidado: Una meta enraizada en la esencia de enfermería. Aquichan. 2013; 13(2): 146147.

12. Izquierdo M. Enfermería: Teoría de Jean Watson y la inteligencia emocional, una visión humana. Rev Cubana Enfermer [Internet]. 2015 [citado 18 dic 2017]; 31(3). Disponible en: http://scielo.sld. $\mathrm{cu} /$ scielo.php?script $=$ sci_arttext\&pi$\mathrm{d}=$ S0864-03192015000300006\&ln$\mathrm{g}=\mathrm{es} \& \mathrm{nrm}=\mathrm{iso} \& \mathrm{t} \operatorname{lng}=\mathrm{es}$

13. Landman C, Canales S, Garay V, García P, López C, Pérez J, et al. Cuidado Humanizado desde la Perspectiva del enfermero de emergencia en un hospital de Quillota, Chile. Enfermería (Montev.). 2014; 3(2): 12-21.

14. Rafael AR. Watson's philosophy, science, and theory of human caring as a conceptual framework for guiding community health nursing practice. ANS Adv Nurs Sci. 2000; 23(2): 34-49.

15. Raile M, Marriner A. Modelos y Teorías en Enfermería. $7^{\text {a }}$ ed. España: Elsevier Mosby; $2011.828 \mathrm{p}$.

16. Watson J, Smith M. Caring science and the science of unitary human beings: A trans-theoretical discourse for nursing knowledge development. J Adv Nurs. 2002; 37(5): 452-61.

17. Poblete M, Valenzuela S. Cuidado Humanizado: un desafío para las enfermeras en los servicios hospitalarios. Cienc. enferm. 2007; XX(4): 499-503.

18. Rotondo de Cassinelli M. Introducción a la bioética. Rev. Urug. Cardiol. [Internet]. 2017 [citado 18 dic 2017]; 32(3): 240248. Disponible en: http://www.scielo. edu.uy/scielo.php?script=sci_arttext\&pi$\mathrm{d}=$ S1688-04202017000300240\&lng=es

19. Perazzo G, Aza C, Mendoza G, Bravo G,
Ramírez S, Mollar E, et al. La deshumanización en la atención profesional: ¿vulnera el derecho a la intimidad? Vida Etica. 2015; 16(1): 7-24.

20. Rodríguez A. Enfermería ante la deshumanización: una necesidad por ser considerada desde la filosofía de Emmanuel Levinas. Enfermería en Costa Rica 2011; 32(1): 37-42.

21. Santacruz-Bravo JD. Humanización de la calidad en la atención clínica en salud desde la perspectiva centrada en el paciente a partir de la Resolución 13437 de 1991. Rev Univ. salud. 2016; 18(2): 373384.

22. Alvis T, Moreno M, Muñoz S. Experiencia de recibir un cuidado de enfermería humanizado en un servicio de hospitalización. [Trabajo de posgrado, especialista en enfermería Cardio-respiratoria] [Bogotá]: Facultad de Enfermería, Universidad Nacional de Colombia; 2001.

23. Rivera L, Triana A. Percepción de comportamientos de cuidado humanizado de enfermería en la Clínica del Country. Av. enferm 2007; 25(1): 56-68.

24. Espinoza L, Huerta K, Pantoja J, Velásquez W, Cubas D, Ramos A. El cuidado humanizado y la percepción del paciente en el Hospital EsSalud Huacho. Octubre de 2010. Cien Des. 2011; 13: 53-61.

25. Gamba NC, Vargas E. Validez de contenido y validez facial del instrumento Percepción de comportamientos de cuidado humanizado. Av. enferm. 2008; 26(2): 7179 .

26. Nunes E, Da Silva LW, Pires EP. La enseñanza superior de enfermería: implicaciones de la formación profesional para el cuidado transpersonal. Rev Lat Am Enfermagem. 2011; 19(2): 252-260.

27. Romero E, Contreras IM, Moncada A. Relación entre cuidado humanizado por enfermería con la hospitalización de pacientes. Hacia promoc. salud. 2016; 21(1): 26-36. 